\title{
Chatter Investigation on Machining of Gray Cast Iron Considering the Damping Effect
}

\author{
$\underline{\text { http://10.3991/ijes.v7i3.11376 }}$ \\ Everton Ruggeri Silva Araújo ( $\square$ ) \\ University of state of Santa Catarina - UDESC, Joinville, Brazil \\ e.ruggeri.eng@gmail.com \\ Giovanni de S. Pinheiro, João Álvaro B. P. Junior, Salomão L. Neto \\ University of Amazônia - UNAMA, Belém, Brazil
}

\begin{abstract}
In recent years, the study of chatter vibrations has been intensifying in the machining of materials. In this paper an investigation of this phenomena was conducted for gray cast iron (CGI). The chatter vibrations in machining process can considerably compromise the workpiece surface finish, tool wear and in some cases provide severe damage to the machine-tool. Thus there is an imminent need to expand the theory of chatter vibrations for the class of brittle materials. To analyze the vibrations of the process of machining and zones where the process is stable, and where it is unstable, the stability lobes diagram was used. This diagram is constructed at low speed cutting, where the phenomenon of damping arises. The damping is a crucial factor in the process, it increases system stability. This effect was considered in the formulation of chatter vibrations using the indentation model of $\mathrm{Wu}$. For experimental validations the signals of cutting force were acquired and analysis was conducted in frequency domain to identify where the vibrations emerged allied with a roughness analysis of the workpiece. The results demonstrated perfectly the consequences of chatter vibrations in surface finish of grey cast iron and proved that the stability lobes diagram provides good results to detect these vibrations, determining the areas where the material removal should be avoid.
\end{abstract}

Keywords-Chatter, Stability Lobes, Machining

\section{Introduction}

Chatter vibration is the one of the types of vibrations existing that has been gaining prominence on the part of the researchers in the area of the machining, due to the numerous problems that this provides, such as poor surface finish of the manufactured parts, excessive tool wear and damages for machine-tool. According to [1] vibrations with excessive amplitudes cause disturbances in the machining process that completely damage the system. [2] states that these vibrations in the machine tool set play an important role for poor productivity in the industry, accelerating the wear of mechanical components, compromising chip formation, causing poor surface finish of the part, and damaging the bearings of the spindle. Conceptually this type of vibration arises 
due to the low structural rigidity of the parts in the machining process associated to a self-excitation mechanism where the process frequencies are close to the natural frequency of the system. With this, chatter lead to the variation in the time of relative positioning between tool and part, causing a phase difference between the cutting teeth, as shown in figure 1.

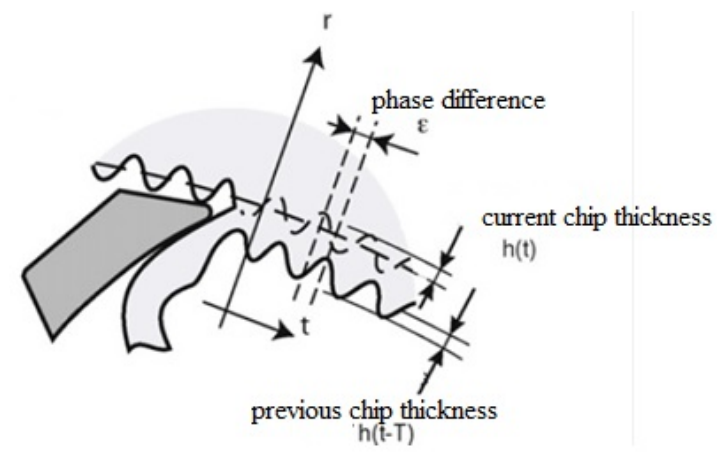

Fig. 1. Phase difference in a multicort process. Source: [5]

In this way, it is necessary to determine the stability conditions of the machining process, verifying areas where there is a stable cut with the absence of these vibrations and where there is an unstable cut with the presence of them.

The determination of the optimal cut condition is usually performed by a twodimensional chart called the stability lobe diagram. This graph is developed numerically by selecting system parameters such as axial depth of cut, machine rotation and modal parameters of the mechanical system. The figure 2 shows an construction of this chart providing different zones on machining where a critical width of cut (bcr) divides these zones, if above, the process is unstable otherwise stable.

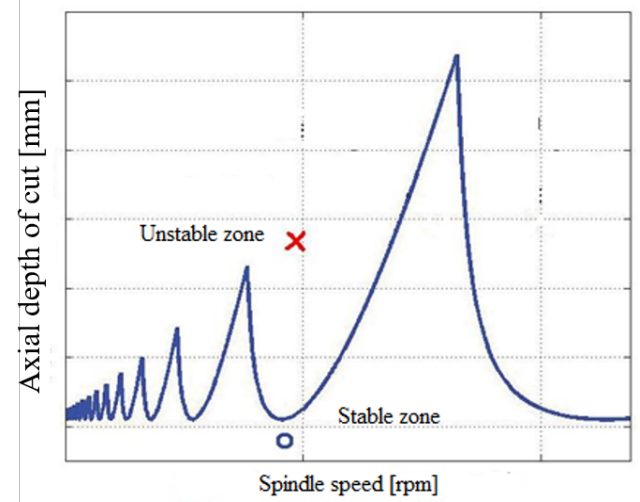

Fig. 2. Stability lobe diagram 
According to [9], the stability lobe diagram is used to optimize the machining process, improving the material removal rate and, consequently, increasing the productivity of a manufacturing process. From this, the conducted tests are used for analysis in processes with high cutting speed, because the lobes created in these conditions have a better spacing between each of them [6].

During machining of materials, the operator can select according to the manufacturer or design the appropriate cutting speed for each manufactured product. When there is a need to process materials at low cutting speeds, the damping phenomenon arises. The damping determines the amplitude of regenerative vibration at the resonance and the time of persistence of the vibration after cessation of its excitation. This feature makes it crucial in material removal processes and process stability [3]. According to [4] apud [7] machining damping occurs at the contact between the tool gap surface with the workpiece when the cutting speed is significantly less than the tip vibration speed the tool. Thus, all these factors make these vibrations allied with the appearance of damping are crucial in the stability study of a process of machining fragile materials with low cutting speed

\section{Mathematical Model of Cutting Forces Considering the Damping Effect}

Based on Kienzle's model for cutting forces, and the damping formulation provides by [8] this paper uses these formulations with the empirical model presented by Altintas for the definition of specific pressure coefficients. In this way considering the mentioned models the equation of this force is expressed in vector form, coupling the part resulting from the damping that arises at low cutting speeds, as presented in (1).

$$
\vec{F}=\vec{K} \cdot b \cdot h\left(\phi_{j}\right)+\vec{C} \cdot b \cdot \frac{\vec{v}}{v_{c}}
$$

Where: $\vec{K}$ vector of specific pressure coefficients., $b$ is the depth of cut, $h\left(\phi_{j}\right)$ instantaneous chip thickness, $\vec{C}$ vector of damping coefficients, $v c$ cutting speed and $\rightarrow$

$v$ vector of chatter velocity .Decomposing the force and coefficients of specific pressure and damping in the tangential, radial and axial directions has:

$$
F_{t}=K_{t} \cdot b \cdot h(\phi)+C_{t} \cdot b \cdot \frac{\vec{v}}{v_{c}}
$$




$$
\begin{aligned}
& F_{r}=K_{r} \cdot b \cdot h(\phi)+C_{r} \cdot b \cdot \frac{\vec{v}}{v_{c}} \\
& F_{a}=K_{a} \cdot b \cdot h(\phi)+C_{a} \cdot b \cdot \frac{\vec{v}}{v_{c}}
\end{aligned}
$$

The instantaneous chip thickness can be represented by equation 2 :

$$
h\left(\phi_{j}\right)=\left\lfloor\Delta x \cdot \operatorname{sen}\left(\phi_{j}\right)+\Delta y \cdot \cos \left(\phi_{j}\right)-\Delta z \cdot \tan (\gamma)\right] \cdot g\left(\phi_{j}\right)
$$

Whereupon: $g\left(\phi_{j}\right)$ is an unit step function, $\gamma$ is the rake angle and $\Delta \mathrm{x}, \Delta \mathrm{y}, \Delta \mathrm{z}$ the variation on position, therefore replacing the equation 2 in 1 for tangencial, radial and axial directions has:

$$
\left\{\begin{array}{l}
F_{t}(j) \\
F_{r}(j) \\
F_{a}(j)
\end{array}\right\}=\left\{\begin{array}{l}
K_{t} \\
K_{r} \\
K_{a}
\end{array}\right\} \cdot b \cdot\left[\operatorname{sen} \phi_{j}, \cos \phi_{j},-\tan \gamma\right] \cdot\left\{\begin{array}{l}
\Delta x \\
\Delta y \\
\Delta z
\end{array}\right\} \cdot g\left(\phi_{j}\right)+\left\{\begin{array}{l}
C_{t} \\
C_{r} \\
C_{a}
\end{array}\right\} \cdot b \cdot \frac{1}{v_{c}} \cdot\left\{\dot{v}_{t}, \dot{v}_{r}, v_{a}\right\}
$$

The specific pressure coefficients can be found using the 4, 4.1 e 4.2 and are divided by specific pressure for shear $K_{c}$ and cutting edge $K_{e}$.

$$
\begin{gathered}
K_{t c}=\frac{4 F_{y c}}{N \cdot a_{p}}, K_{t e}=\frac{\pi F_{y e}}{N \cdot a_{p}} \\
K_{r c}=-\frac{4 F_{x e}}{N \cdot a_{p}}, K_{r e}=-\frac{\pi F_{x e}}{N \cdot a_{p}} \\
K_{a c}=\frac{\pi F_{z c}}{N \cdot a_{p}}, K_{a e}=\frac{2 F_{z e}}{N \cdot a_{p}}
\end{gathered}
$$

Where: $\mathrm{N}$ is the number of teeths and $A p$ na axial depth of cut. The damping coefficients can be estimated by equations $5,5.1$ e 5.2 .

$$
\begin{gathered}
C_{t}=\mu \cdot K s p \cdot C d \cdot\left[1+\frac{L}{b}\right] \\
C_{r}=K s p \cdot C d \cdot \operatorname{sen} \alpha
\end{gathered}
$$




$$
C_{a}=K s p \cdot C d \cdot\left[\cos \alpha+\frac{L}{b}\right]
$$

Which $K s p$ is the specific damping coefficient express by equation 6 .

$$
K s p=\frac{E}{1,29 \cdot \rho \cdot(1-2 v)}
$$

In this way the force being a part referring to the regenerative part plus the damping share and realizing a change of the system of local coordinates of the expressions to a system of global coordinates, we create the matrices [A] of displacement coefficients dynamics and $[\mathrm{B}]$ coefficients of dynamic damping resulting in equation 7.

$$
\left\{\begin{array}{l}
F_{x} \\
F_{y} \\
F_{z}
\end{array}\right\}=b \cdot[A] \cdot\left\{\begin{array}{l}
\Delta_{x} \\
\Delta_{y} \\
\Delta_{z}
\end{array}\right\}+\frac{b}{v_{c}} \cdot[B] \cdot\left\{\begin{array}{l}
\dot{x} \\
y \\
\dot{z}
\end{array}\right\}
$$

And representing the self-excited part of the force, in the time domain, results in:

$$
\vec{F}(t)=b \cdot[A](t) \cdot \Delta(t)+\frac{b}{v_{c}} \cdot[B](t) \cdot \vec{v}(t)
$$

Thus, by using the mean expansion of the Fourier series for elimination in the dependent term term and the Laplace transform one can obtain the eigenvalue problems and construct the stability lobe diagram.

$$
\vec{F}(s)=b \cdot\left[A_{0}\right] \cdot\left(1-e^{-s T p}\right) \cdot \vec{v}(s)+s \cdot \frac{b}{v_{c}} \cdot\left[B_{0}\right] \cdot \vec{v}(s)
$$

With the vibration vector expressed by the product of the transfer function for structure $(\mathrm{G}(\mathrm{s}))$ and force vector

$$
\vec{v}(s)=G(s) \cdot \vec{F}(s)
$$

Replacing the equation 9 into equation 10, an eigenvalue problem denoted by: 


$$
\left\{[I]-b \cdot\left[A_{0}\right] \cdot\left(1-e^{s T}\right) \cdot[G(s)] \cdot\left[[I]-s \cdot \frac{b}{v_{c}} \cdot\left[B_{0}\right] \cdot[G(s)]\right]^{-1}\right\} \cdot F(s)=\{0\}
$$

The stability of the system is analyzed on frequency domain with $\mathrm{s}=\mathrm{i} \omega \mathrm{c}$ where $\omega \mathrm{c}$ is the chatter frequency

$$
\left\{[I]-b \cdot\left[A_{0}\right] \cdot\left(1-e^{i \omega c \cdot T}\right) \cdot[G(i \omega c)] \cdot\left[[I]-i \omega c \cdot \frac{b}{v_{c}} \cdot\left[B_{0}\right] \cdot[G(i \omega c)]\right]^{-1}\right\} \cdot F(i \omega c)=\{0\}
$$

In an iterative way due to the damping term the characteristic equation becomes:

$$
\left[[I]+\Lambda[\phi(i \omega c)] \cdot C_{0}\right]
$$

Being $\mathrm{C} 0$ the term referring to the damping process in the frequency chatter vibration equal to:

$$
C_{0}=\left[[I]-i \omega c \cdot \frac{b}{v_{c}} \cdot\left[B_{0}\right] \cdot[G(i \omega c)]\right]^{-1}
$$

\section{$3 \quad$ Materials and Methods}

In order to perform the experimental procedure of this paper, a device was designed with the purpose of simplifying the model for one degree of freedom, considering the effect of vibration only in the $\mathrm{Z}$ axis as shown in figure 3 .
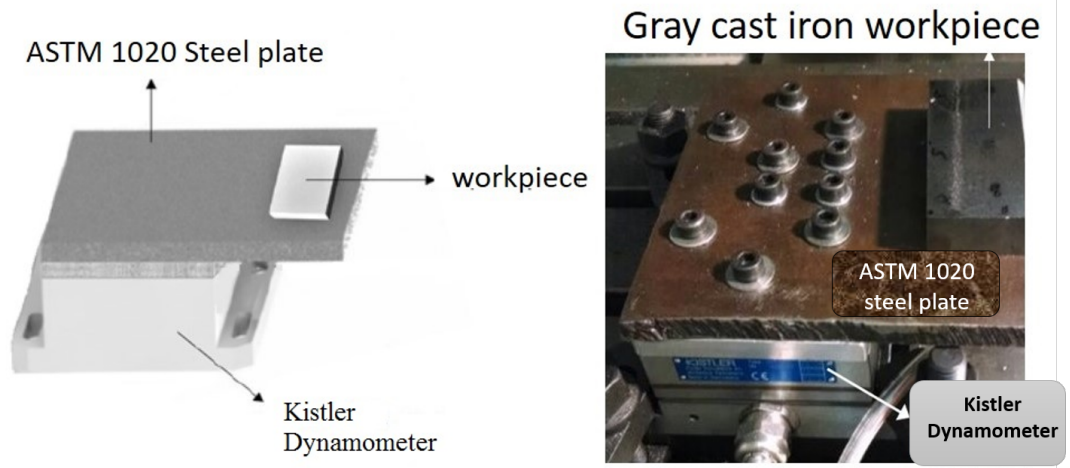

Fig. 3. Built device for experiments 
The system has the similar behavior of a cantilever plate, in which along its length the structural rigidity changes, being maximum next to the crimping and decreasing toward to the free end. The objective to construct this device is reducing the degree of freedom for the system. In order to obtain the modal system parameters an accelerometer is attached to the workpiece and its signal is measured and analyzed.

The machined material was ASTM FC60 gray cast iron with a Coromill R345 tool made by with $45^{\circ}$ tooth position angle $(\chi \mathrm{r})$. The milling was conducted in a ROMI D600 machining center of university of the state of Santa Catarina. Four trials were performed to analyze the chatter vibration, varying the axial cutting depth (ap), according to the critical width of cut (bcr) founded in stability lobes. Two rotational regions of the spindle were analyzed, one in the range of 1500 to $1700 \mathrm{rpm}$ and another in the range of 800 to $900 \mathrm{rpm}$ booth with low cutting speed. Table 1 shows the cutoff parameters used in each experiment.

Table 1. Cutting parameters for tests

\begin{tabular}{|l|c|c|c|c|}
\hline \multicolumn{1}{|c|}{ Tests } & Axial depht of cut $(\boldsymbol{a p})$ & $\begin{array}{c}\text { Critical width of cut } \\
(\boldsymbol{b} \boldsymbol{c r})\end{array}$ & Cutting velocity (vc) & $\begin{array}{c}\text { Spindle speed } \\
(\boldsymbol{n})\end{array}$ \\
\hline Assay 1 & $4,5 \mathrm{~mm}$ & $4,2 \mathrm{~mm}$ & $397 \mathrm{~m} / \mathrm{min}$ & $1580 \mathrm{rpm}$ \\
\hline Assay 2 & $2,5 \mathrm{~mm}$ & $3,9 \mathrm{~mm}$ & $411 \mathrm{~m} / \mathrm{min}$ & $1640 \mathrm{rpm}$ \\
\hline Assay 3 & $1,5 \mathrm{~mm}$ & $1,3 \mathrm{~mm}$ & $203 \mathrm{~m} / \mathrm{min}$ & $809 \mathrm{rpm}$ \\
\hline Assay 4 & $1,1 \mathrm{~mm}$ & $1,3 \mathrm{~mm}$ & $215 \mathrm{~m} / \mathrm{min}$ & $855 \mathrm{rpm}$ \\
\hline
\end{tabular}

The cutting parameters were kept constant during all experiments was showed in Table 2.

Table 2. Cutting parameters kept constants

\begin{tabular}{|l|l|}
\hline Feed rate Per Tooth (fz) & $0,015 \mathrm{~mm} / \mathrm{rot}$ \\
\hline Radial Depth of cut (Ae) & $13 \mathrm{~mm}$ \\
\hline Numbers of teeth & 6 \\
\hline Tool diameter & $80 \mathrm{~mm}$ \\
\hline Sense & discordant \\
\hline Insert Type & $345 \mathrm{R}-1305 \mathrm{MKM}$ \\
\hline Modal Parameters: Naturals frequencies & $\begin{array}{l}\text { Assay 1:606 Hz, assay 2: } 607 \mathrm{~Hz}, \text { Assay 3: } 586 \mathrm{~Hz}, \text { Assay 4: } 594 \\
\mathrm{~Hz}\end{array}$ \\
\hline
\end{tabular}

All experiments were performed at low cutting speeds, which, the settlement state makes damping very significant for machining. To model the specific damping coefficient the model of [8] was applied. Which the depth of plastic deformation was estimated at $0.70 \mathrm{~mm}$ the gray cast iron module (E) was $110 \mathrm{GPa}$ and the Poisson radius (v) was 0.26 . The wear width was constant throughout the insert with a value of $0.08 \mathrm{~mm}$. These tool holders have a length of $2 \mathrm{~mm}$ and angle of tool-tooth positioning is $45^{\circ}$.

In the experiments, the critical width of cut (bcr) was estimated from the stability lobe diagram technique developed in the 1960 s by Tobias and improved by several authors over the time. 
The machining force produced by the material removal process was purchased by a Kistler model 9257B piezoelectric ring and processed by LABVIEW software. Matlab helps to obtain a FFT power spectrum of the force signal. The measuring range of the piezoelectric platform corresponded from $-5 \mathrm{kN}$ to $10 \mathrm{kN}$ in the $\mathrm{x}, \mathrm{y}$ and $\mathrm{z}$ axis of the dynamometer. The platform strength signals were processed using a National Instruments NI-6001 USB module where the acquisition rate of the machining force signal was 10,000 points / second.

Therefore the experimental tests depending of stability lobe diagram constructed in Matlab software to verify the effects of the vibrations in the machining process. After this procedure an roughness analysis was conducted using Mitutoyo SJ-210 digital rugosimeter capable of measuring geometrical imperfections up to $17.5 \mathrm{~mm}$ and $\mathrm{z}$ axis up to $360 \mu \mathrm{m}$. (figure 4 )

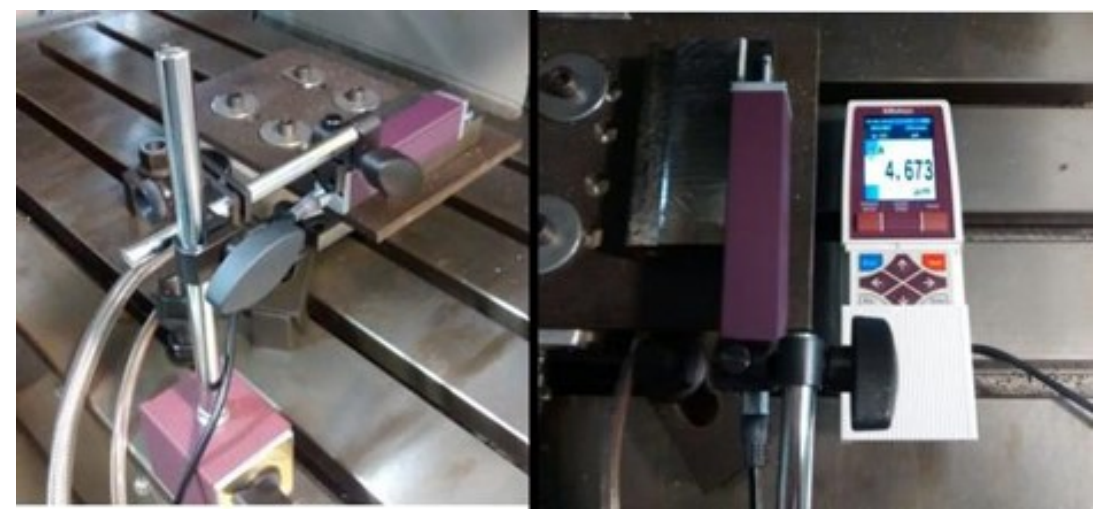

Fig. 4. Roughness method of analysis used after experiments

This step of the adopted methodology was applied to verify the correlation of the system stability in the produced surface finish.

\section{$4 \quad$ Results and Discussion}

In order to validate the stability lobes diagram, the tests were conducted under different cutting conditions using the test force signals from kistler dynamometer to prove the occurrence of chatter vibrations To accomplish this the Fast Fourier Transmission (FFT) was used to analyze the signal of force in frequency domain to detect whether there will be vibration peaks near to the peaks of the natural frequency of the system.

Figure 5 and 9 show the stability lobes obtained for Experiments 1, 23 and 4, from them a previous behavior of the system can be found choosing the axial depth of cut above or below to the critical width of cut. By means of this technique and considering the damping effect, the experiment one was planned for a situation of instability on machining with the applied axial depth of cut $(a p=4,5 \mathrm{~mm})$ was above the critical width of cut $(b c r=4,2 \mathrm{~mm})$. For test two the experiment expected a stable cutting for 
the reason that the axial depth of cut was lower than the critical depth of cut $(a p=2,5$ $\mathrm{mm}<b c r=3,9 \mathrm{~mm}$ ). (see figure 5)

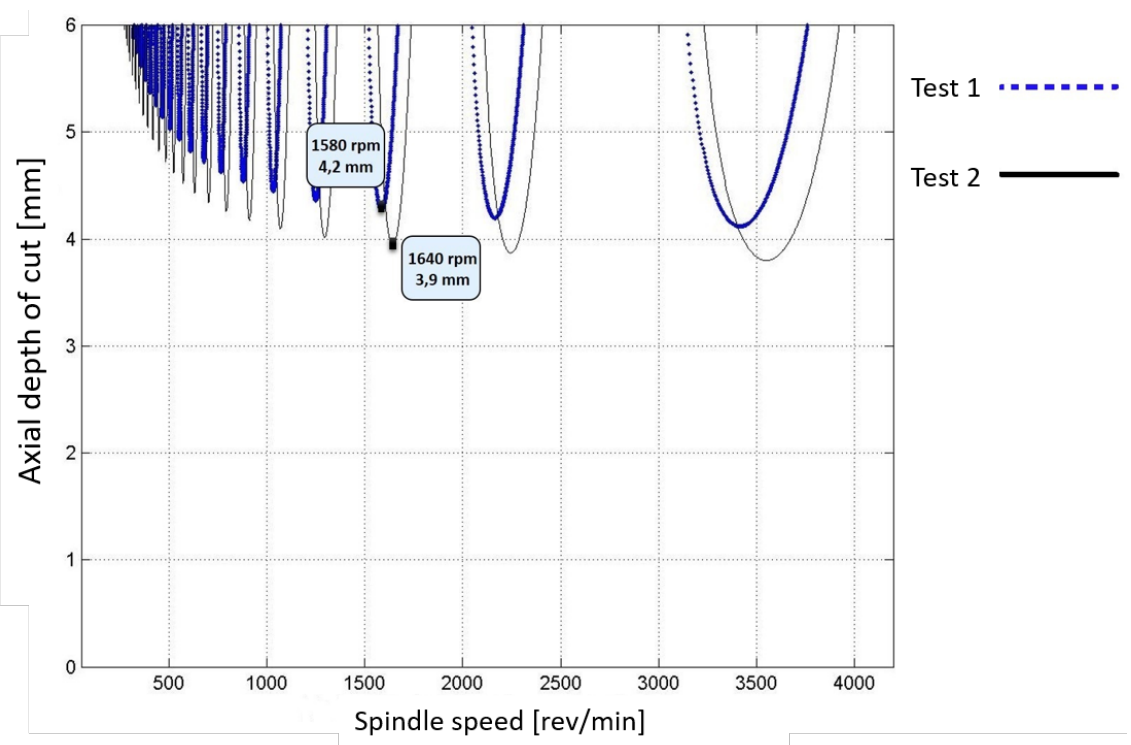

Fig. 5. Stability lobes diagram for experiments 1 and 2.

Using the FFT power spectrum of force for tests 1 and 2, was verified a frequency of $612 \mathrm{~Hz}$ with an amplitude equal to $4,1 \times 10^{4} \mathrm{~N}$ is modulated nearest from the natural frequency of system corresponding to $606 \mathrm{~Hz}$, this behavior implies that in this experiment the chatter vibration act making the system unstable, which is will compromise the tool, the workpiece surface finish and the machine-tool.

The FFT signal for experiment 2 does not has frequencies nearest of the natural frequency, besides, the amplitude of $612 \mathrm{~Hz}$ frequency founded in experiment 1 decays. This behavior is a prove that in this assay will be a stable cut. The frequencies founded of $158 \mathrm{~Hz}$ and $316 \mathrm{~Hz}$ are harmonics due to the initial contact of the teeth of the tool in the workpiece. Figure 6 shows the FFT for signal force. 


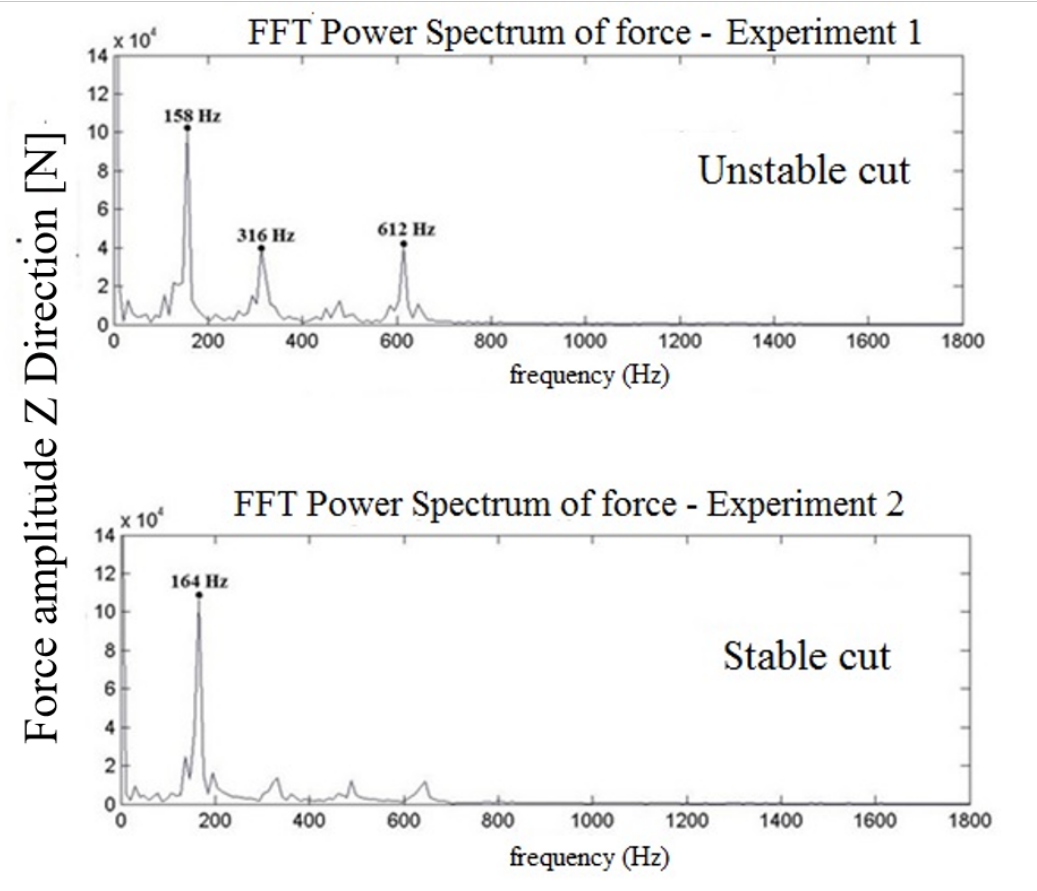

Fig. 6. FFT power spectrum of experiments 1,2

Accordingly, with the results from FFT spectral signal the stability lobes diagram proves that this techinique is effective to detect the chatter vibration. Figure 7 shows the workpiece after the machining
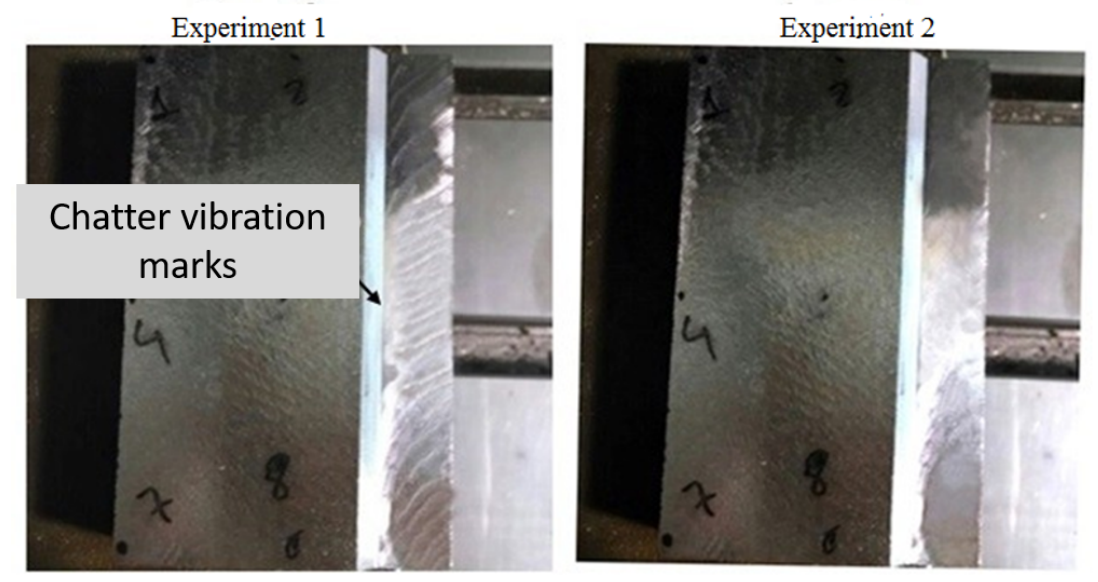

Fig. 7. Workpiece after milling of gray cast iron. 
The presence of chatter vibrations also had an impact on the surface finish of the part, with figure 8 showing this parameter from the average roughness (Ra) and total roughness $(\mathrm{Rz})$. Test 1 had a total roughness $\mathrm{Rz}$ of $46.5 \mu \mathrm{m}$ and average roughness $\mathrm{Ra}$ in the value of $9.2 \mu \mathrm{m}$. This poor surface finish effect results from the appearance of chatter vibration in the process and greater removal of material. From the point of view of the vibration peaks it is proven when compared to test 2 where a total $\mathrm{Rz}$ roughness of $9.9 \mu \mathrm{m}$ and average roughness $\mathrm{Ra}$ of $1.3 \mu \mathrm{m}$ was obtained.

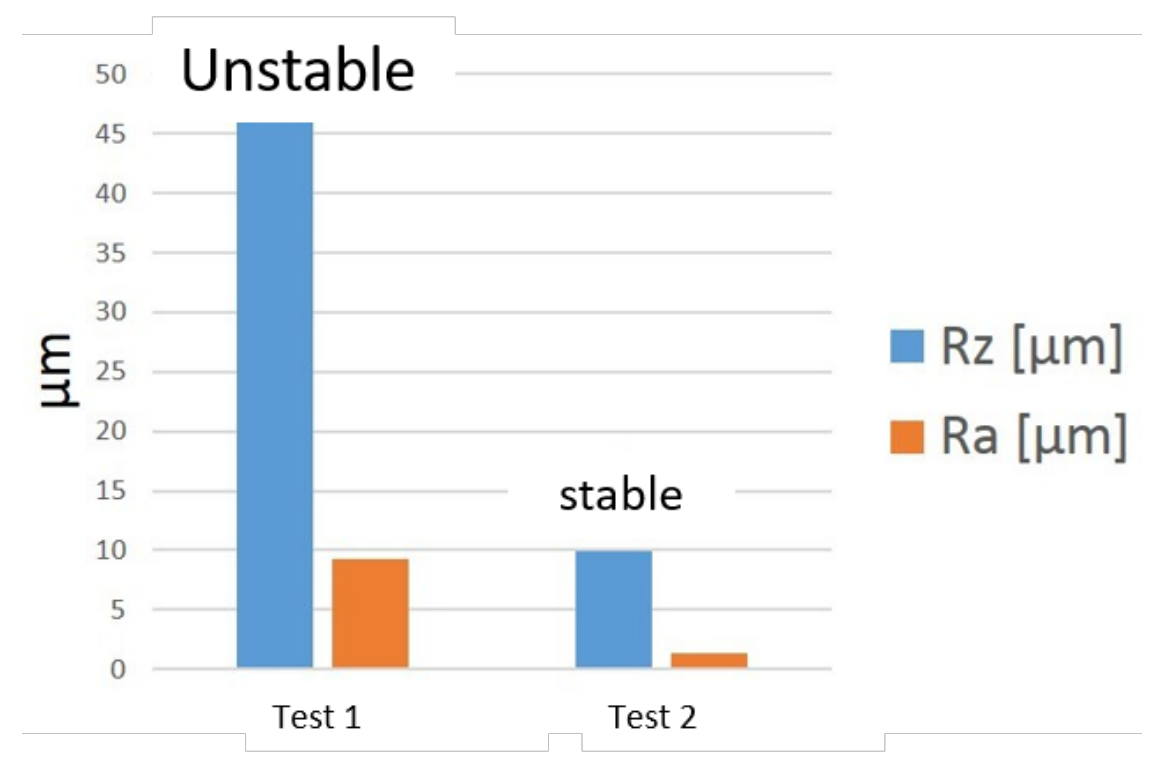

Fig. 8. Roughness tests 1 and 2 .

Test 3 was conducted at $809 \mathrm{rpm}$ and axial depth of cut above critical depth, 1.5 $\mathrm{mm}$. The natural frequency of the system was $586 \mathrm{~Hz}$, the frequency of tooth passage was $80.9 \mathrm{~Hz}$.

Test 4 was conducted at $855 \mathrm{rpm}$, axial depth of cut below the stability limit of 1.1 $\mathrm{mm}$. The natural frequency of the system was identified at $594 \mathrm{~Hz}$, frequency of tooth passage was $85.5 \mathrm{~Hz}$. Figure 9 shows the stability lobe diagram for these tests. 


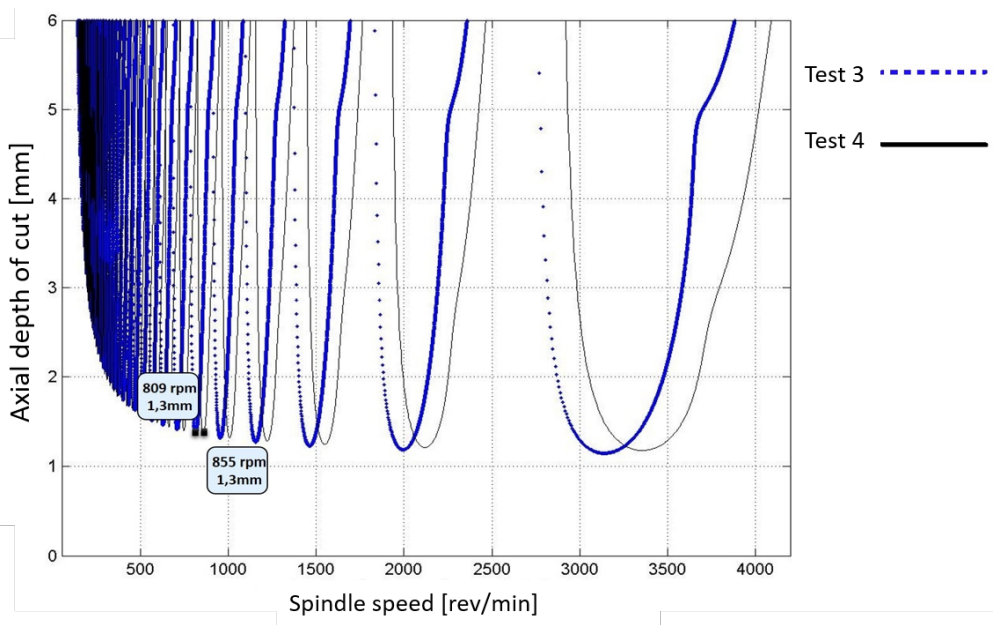

Fig. 9. Stability lobes diagram for experiments 3 and 4.

From the signal of the FFT of force also verified multiple frequencies very close to the natural frequency of the system in the values of $586 \mathrm{~Hz}$ and $605 \mathrm{~Hz}$ with amplitude of $2.1 \times 104 \mathrm{~N}$ and $1.6 \times 104 \mathrm{~N}$ respectively. The input frequency of the teeth was $80 \mathrm{~Hz}$ for test 3 with its first harmonic scoring at $160 \mathrm{~Hz}$. As a result, an unstable cut was validated. For the test 4 the frequency of the teeth input was $85 \mathrm{~Hz}$ and a considered stable cut is verified without the presence of frequency peaks near the natural frequency of $594 \mathrm{~Hz}$. Figure 10 shows the strength signal spectrum of these assays showing such frequency peaks.

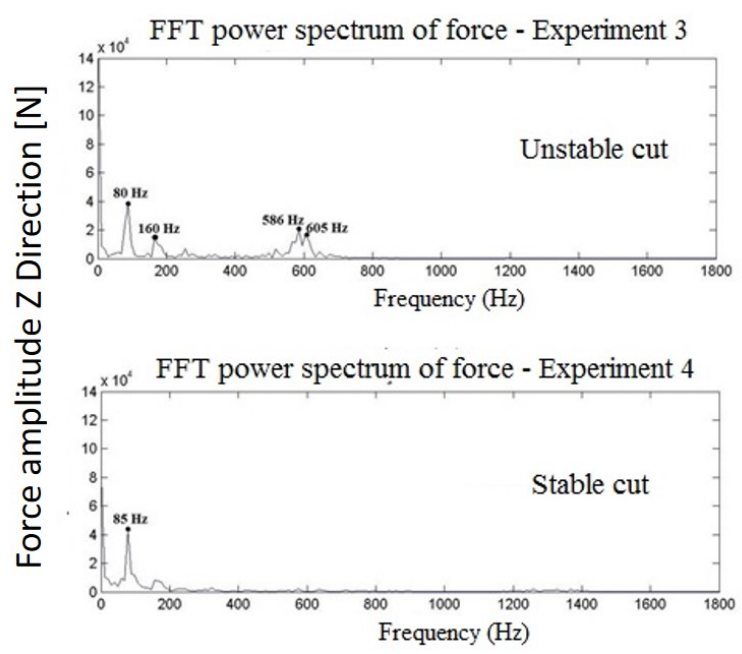

Fig. 10.FFT power spectrum of experiments 1,2 . 
Thus, the experiments correctly validated the simulated lobes, and as a consequence of the emergence of chatter vibration, there was an apparent difference between the two tests observed on the surface of the specimens. (Figure 11)

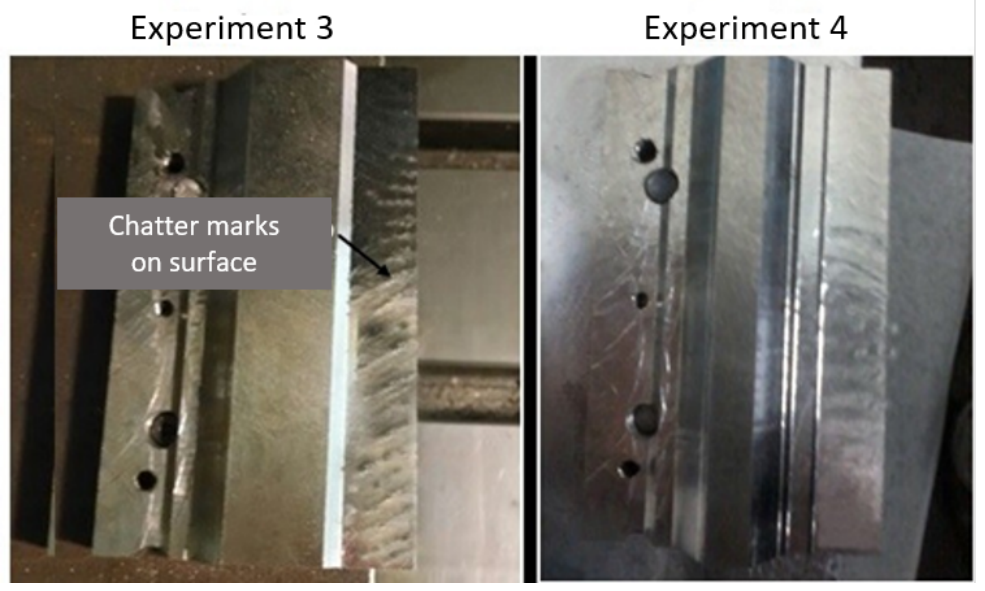

Fig. 11.Workpiece after milling of gray cast iron

This effect could also be observed in the finishing of the parts by roughness parameters. For test 3 the total roughness $\mathrm{Rz}$ value found was $16.6 \mu \mathrm{m}$ and $2.8 \mu \mathrm{m}$ for the average roughness Ra against $13.9 \mu \mathrm{m} \mathrm{Rz}$ and $2.4 \mu \mathrm{m} \mathrm{Ra}$ in assay 4 as shown in figure 12.

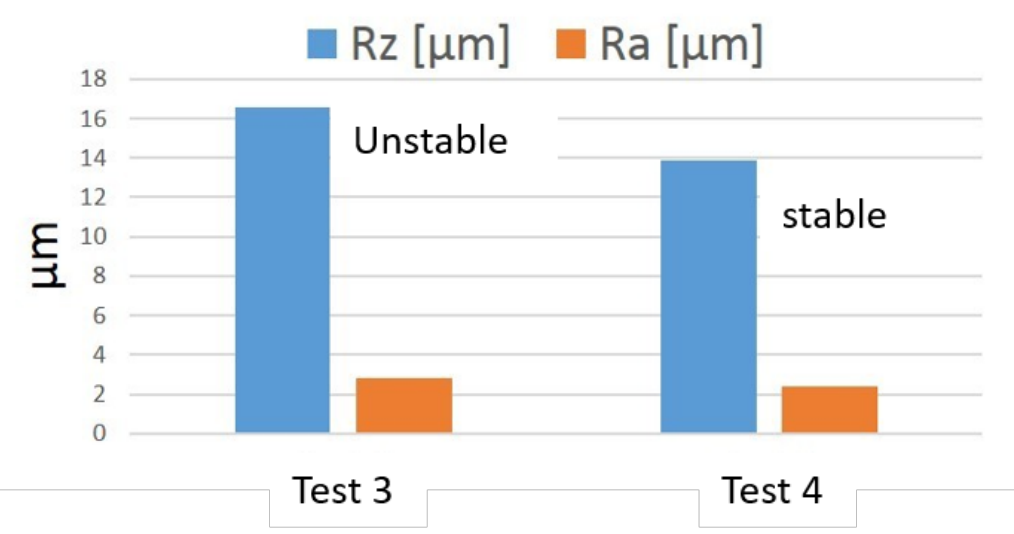

Fig. 12.Roughness in tests 3 and 4 . 


\section{Conclusion}

The construction of the stability lobe diagram is a good palliative measure to control the appearance of chatter vibrations in gray cast iron milling. The damping effect tends to be more effective in regions of machining with low shear rates, which causes in the stability lobe diagram an effect that tends to reduce the spacing between the lobes. The machining test considering this effect must to be carefully planned to avoid these types of vibrations under conditions in these regions. From the obtained results a good efficacy of the diagram to predict the chatter vibrations was founded and enabled the consequences in the surface finish of workpiece. The parameters chosen for the tests, stooded at the low cutting region of machining, in this region the instability and damping condition impacted on the surface finish, where through tests 1 and 3 the roughness was higher than tests 2 and 4 planned to be a stable experiment. In this way, it is necessary to plan carefully the tests on gray cast iron at low speeds with the highest accuracy since the influence of damping is crucial in these regions.

\section{$6 \quad$ References}

[1] Altintas, Y. (2000). "Manufacturing Automation: Metal Cutting Mechanics, Machine tool vibrations and CNC Design". s.1.: Cambridge University Press. https://doi.org/10.1017 /cbo9780511843723.006

[2] Altintas, Y.; Budak, E.(1998). “Analytical prediction of chatter stability in milling — part i: general formulation", Journal of Dynamic Systems Measurement and Control 120, pp.2230. https://doi.org/10.1115/1.2801317

[3] Huang, C. Y.; Wang Junz, J.-J. (2011). "Effects of cutting conditions on dynamic cutting factor and process damping in milling". International Journal of Machine Tools \& Manufacture, v.51, pp.320-330. https://doi.org/10.1016/j.ijmachtools.2010.12.004

[4] Jin, X. (2012) "Mechanics and dynamics of micro-cutting process". Doctor Thesis, University of British Columbia. Vancouver, Canadá.

[5] Rahnama, ramin; sajjadi, mozhdeh; park, simon. S. (2009) "Chatter suppression in micro end milling with process damping”. Journal of materials processing technology, v.209, pp.5766-5776. https://doi.org/10.1016/j.jmatprotec.2009.06.009

[6] Tyler, C. (2012) "Process damping analytical stability analysis and validation". Dissertação de Mestrado. Universidade da Carolina do norte em Charlotte.

[7] Wallace, P.W.; Andrew, C. (1965). "Machining forces: some effects of tool vibration”. J. Mech Eng Sci. 7(2): 152-162

[8] Wu, D. W. (1989) "A new approach of formulation the transfer function for dynamic cutting process". Journal of Engineering for Industry. v.111, p.37-47

[9] Yue, J. (2006) “Creating a stability lobe diagram.” International Journal of Modern Engineering. INTERTECH. Conference. Kean University, USA. 


\section{$7 \quad$ Authors}

Everton Ruggeri Silva Araújo is a professor of University of Amazônia:UNAMA and was a master student and machining researcher from university of the state of Santa Catarina: UDESC. (e-mail: e.ruggeri.eng@gmail.com)

Giovanni de S. Pinheiro is a is a professor of University of Amazônia:UNAMA and researcher of the acoustic and vibration lab from UFPA. (e-mail: giovannipinheiro@yahoo.com.br)

João Álvaro B. P. Junior is a bachelor's student of mechanical engineering from University of Amazônia:UNAMA (e-mail: alvarobpj8@gmail.com)

Salomão L. Neto is a bachelor's student of mechanical engineering from University of Amazônia:UNAMA (e-mail: salomaolevy@hotmail.com)

Article submitted 2019-07-25. Resubmitted 2019-08-20. Final acceptance 2019-08-21. Final version published as submitted by the authors. 